\title{
Atopic Dermatitis: The New Therapeutic Revolution in Dermatology
}

\section{Dermatite Atópica: A Nova Revolução Terapêutica na Dermatologia}

Tiago TORRES $\square^{1,2}$

Acta Med Port 2017 Oct;30(10):669-670 - https://doi.org/10.20344/amp.9787

Keywords: Dermatitis, Atopic/physiopathology; Dermatitis, Atopic/therapy; Molecular Targeted Therapy

Palavras-chave: Dermatite Atópica/fisiopatologia; Dermatite Atópica/tratamento; Terapia de Alvo Molecular

Biologic agents revolutionized the management of psoriasis over the past decade, leading to a significant shift in the treatment of this chronic disorder that invariably requires long-term therapy to maintain clinical response. ${ }^{1}$ This revolution was mainly due to the major advances made in the past 30 years in elucidating the pathogenesis of psoriasis: first identifying the role of TNF and later the key role of IL-23/IL-17 axis in psoriatic inflammation. ${ }^{2} \mathrm{~A}$ similar revolution is currently being observed in atopic dermatitis (AD), as a growing understanding of the molecular mechanisms that contribute to $A D$ is enabling the development of new targeted therapies.

$A D$ is a common, chronic and relapsing inflammatory skin disorder with an increasing incidence during the past few decades, especially in developed countries. ${ }^{3}$ It is associated with significant morbidity and quality of life impairment, and recent studies have demonstrated a burden of disease comparable to other chronic conditions like diabetes mellitus and cystic fibrosis. ${ }^{4}$ Active disease has major psychosocial and financial implications for individuals, their families, health care providers, and society as a whole. Moreover, $A D$ has been put forward as a systemic disorder associated with increased risk of various medical and psychiatric comorbidities, just like psoriasis, although in a lesser degree. ${ }^{5}$

Contrary to psoriasis, the treatment of moderate-tosevere $A D$ remained challenging and limited. Cyclosporine is the only systemic agent approved for the treatment of $A D$ in many European countries and alternative off-label options include methotrexate, azathioprine and mycophenolatemofetil, with similar response rates, but limited by their safety profile. Thus, the need for novel, efficacious, safe and targeted treatments is at its greatest.

The pathogenesis of $A D$ is complex and combines barrier dysfunction, cutaneous and systemic immune dysregulation and the role of microbiome. ${ }^{6}$ During the past decade, the underlying molecular basis for $A D$ has been increasingly understood as well as for pruritus, both at the peripheral and central nervous system levels. $A D$ is characterized by skin epidermal barrier disruption which leads to chronic inflammation with epidermal hyperplasia and cellular infiltrates, including T-cells, dendritic cells, eosinophils, and type-2 T-helper cell (Th2). ${ }^{6}$ Regarding immune dysregulation, $A D$ is currently considered a biphasic or combined T cell-mediated disease. A Th2 signal predominates in the acute phase, secreting IL-4, IL-5, IL13, IL-25, and IL-31, whereas a Th2-Th1 switch promotes disease chronicity. Moreover, a Th22 cells secreting IL-22, and in a smaller degree, Th17 cells secreting IL-17 play a role in the initiation and maintenance of AD. ${ }^{6}$ Finally, although IgE has been considered a hallmark of atopic diseases, including $A D, \lg E$ itself is not a key mediator of AD pathogenesis. ${ }^{6}$

This growing understanding of the mechanism for $A D$, particularly focused on suppressing the skewed immune activation, is leading to an expanding pipeline of new and targeted topical and systemic therapies. In the past year, the first topical PDE4 inhibitor (crisaborole) and the first effective subcutaneously administered biologic (dupilumab) targeting the IL-4/IL-13 receptor, that received the 'breakthrough therapy' designation of and priority review by the US Food and Drug Administration (FDA), have become available for mild-to-moderate and moderateto-severe $A D$, respectively. ${ }^{7,8}$ Several other topical, oral and injectable therapies are currently being studied in AD. Pitrakinra specifically blocks IL-4, while tralokinumab and lebrikizumab selectively targets IL-13. Nemolizumab, an IL31 receptor inhibitor, is the first biologic specifically targeting IL-31, also known as the 'pruritus cytokine', and has shown to significantly inhibit pruritus in the phase two clinical trial. As expected, the anti-IgE biologic omalizumab did not show efficacy in clinical trials. ${ }^{8,9}$

Janus kinases (JAK) inhibitors are another promising therapeutic class for the treatment of AD. JAK-STAT (Janus kinase - signal transducer and activator of transcription) is an intracellular signaling pathway on which many different proinflammatory cytokines (including IL-4, IL-5, IL-13, and IL-31) elicit their pathophysiologic functions. JAK inhibitors are broad-acting small molecules for oral or topical administration, with anti-inflammatory and

1. Serviço de Dermatologia. Centro Hospitalar e Universitário do Porto. Porto. Portugal.

2. Mestrado Integrado em Medicina. Instituto de Ciências Biomédicas Abel Salazar. Universidade do Porto. Porto. Portugal.

$\triangle$ Autor correspondente: Tiago Torres. torres.tiago@outlook.com

Recebido: 13 de outubro de 2017 - Aceite: 25 de outubro de 2017 | Copyright $\odot$ Ordem dos Médicos 2017 
anti-proliferative activity. Oral baricitinib (JAK1/2 inhibitor; phase two completed), PF-04965842 (JAK1 inhibitor; in phase two) and upadacitinib (JAK1 inhibitor; in phase two) are currently being evaluated in AD, while INCB18424 (JAK1/2 inhibitor) and LEO 124249/JTE-052 (Pan-JAK inhibitor; in trials in Japan), are in phase two clinical trials for topical administration. , $^{8,9}$ Large, randomized, placebocontrolled clinical trials are eagerly waited to clarify the potential benefit of JAK inhibitors in AD patients.

Many of these therapies are also being investigated in other diseases, such as asthma, chronic obstructive pulmonary disease or allergic disorders. Thus, just like it happened in psoriasis, another successful therapeutic transfer from dermatology to other medical areas may be repeated.

We are living exciting times in AD. Several mechanistic studies are now allowing the identification of key mediators and mechanisms that drive and perpetuate $A D$, leading to the development of several new and targeted therapies,

\section{REFERENCES}

1. Torres T, Velho GC, Sanches M, Selores M. Psoriasis in the era of biologics. Acta Med Port. 2010;23:493-8.

2. Torres T, Filipe P. Interleukin-17 as a therapeutic target in psoriasis. Acta Med Port. 2014;27:252-8.

3. Bieber T. How to define atopic dermatitis? Dermatol Clin. 2017;35:27581.

4. Drucker AM, Wang AR, Qureshi AA. Research gaps in quality of life and economic burden of atopic dermatitis: the National Eczema Association burden of disease audit. JAMA Dermatol. 2016;152:873-4.

5. Brunner PM, Silverberg JI, Guttman-Yassky E, Paller AS, Kabashima K, Amagai $\mathrm{M}$, et al. Increasing comorbidities suggest that atopic dermatitis revitalizing this previously stagnant field, that will change dermatological praxis profoundly and will increase physicians' ability to help to improve the patients' lives. However, despite overall confidence we still have to be aware of unexpected risks. Although the side effect profile of these new drugs appears safe, long-term effects are still unclear.

Researchers and dermatologists must now focus on better defining different subtypes of $A D$, being able to early identify those patients which are in the need of a maximum treatment. These new directed therapies will change current algorithms of care and its careful use will allow practitioners to provide optimal therapy while minimizing adverse impacts on safety and cost.

\section{CONFLICTS OF INTEREST}

Tiago Torres has received honoraria for acting as a consultant and/or as a speaker at events sponsored by AbbVie, Celgene, Janssen, Leo-Pharma, Lilly-Eli and Pfizer.

is a systemic disorder. J Invest Dermatol. 2017;137:18-25.

6. Malik K, Heitmiller KD, Czarnowicki T. An update on the pathophysiology of atopic dermatitis. Dermatol Clin. 2017;35:317-26.

7. Udkoff J, Waldman A, Ahluwalia J, Borok J, Eichenfield LF. Current and emerging topical therapies for atopic dermatitis. Clin Dermatol. 2017;35:375-82.

8. Simpson E, Udkoff J, Borok J, Tom W, Beck L, Eichenfield LF. Atopic dermatitis: emerging therapies. Semin Cutan Med Surg. 2017;36:12430.

9. Renert-Yuval Y, Guttman-Yassky E. Systemic therapies in atopic dermatitis: the pipeline. Clin Dermatol. 2017;35:387-97. 